\title{
Non-Imaging Optics for Improving Waste Heat Collection with Thermoelectrics
}

\author{
Zeming He, ${ }^{1,2 \dagger}$ Moo-Xin Foo, ${ }^{3, \dagger}$ Derrick Yong, ${ }^{3, \dagger}$ Ting Ma, ${ }^{4}$ Yong Hao, ${ }^{1,2}$ Hang Zhang ${ }^{1,{ }^{*}}$ and Ding Ding ${ }^{5^{*}}$
}

Waste heat is often dumped into the environment and there have not been many cost-effective ways so far to harness it especially if it is of a low grade. Thermal radiation is the predominant form of heat transport between Space and Earth but it is often not considered to be efficient for waste heat applications. Here, we utilize the concept of non-imaging optics to enhance the capture of waste heat from a hot object in the form of thermal radiation. We designed and fabricated a compound parabolic concentrator (CPC) that is purely reflective in the mid-infrared using geometric non-imaging optics. With the CPC present, in between a hot plate and a target separated $10 \mathrm{~cm}$ apart under ambient conditions, we were able to increase the temperature of the target by as much as $20{ }^{\circ} \mathrm{C}$. At the same time, our simulations and experiment demonstrate remarkable improvement in thermoelectric (TE) efficiency and output power compared to a bare TE exposed to the same radiative heat flux. Our work serves as a proof-of-concept for demonstrating the potential to collect waste heat remotely using pure thermal radiation.

Keywords: Waste heat; Non-imaging optics; Thermoelectrics

Received 30 August 2019, Accepted 19 October 2019

DOI: $10.30919 /$ esee 8 c335

\section{Introduction}

We are generating more waste heat each day from burning of fossil fuel but these waste heat are frequently discharged into the environment. There exist various grades of waste heat depending on the temperature of heating process. High grade waste heat, which are above $600{ }^{\circ} \mathrm{C},{ }^{1}$ can be used for other heating processes due to their high temperature. Often, waste heat recovery using heat exchangers or thermoelectrics can turn high grade waste heat into electricity. ${ }^{2}$ However, for medium to low grade waste heat which are less than $600{ }^{\circ} \mathrm{C}$, it is often not efficient or cost-effective to convert them into other forms of energy using heat exchangers or thermoelectrics. ${ }^{3}$ Thus, a lot of times low-grade waste heat is discharged into the environment.

Compared to conduction and convection used in heat exchangers, thermal radiation has been viewed as the most inefficient way to transfer heat between two objects. This is in part due to the difficulty in controlling the heat transfer as thermal radiation does not require a medium. Recent advances in optics and photonics have enabled the use of thermal radiation to effectively control heat transport. ${ }^{47}$ However,

'Institute of Engineering Thermophysics, Chinese Academy of Sciences, Beijing, China

${ }^{2}$ University of Chinese Academy of Sciences, Beijing 100049, China

${ }^{3}$ Singapore Institute of Manufacturing Technology, 2 Fusionopolis Way, Innovis \#08-04, Singapore

${ }^{4}$ Key Laboratory of Thermo-Fluid Science and Engineering, MOE, Xi'an Jiaotong University, Xi'an, Shaanxi, 710049, China

${ }^{5}$ Institute of Material Research and Engineering, 2 Fusionopolis Way, Innovis \#08-03, Singapore

*E-mail: zhanghang@iet.cn; Ding_Ding@imre.a-star.edu.sg

'These authors contributed equally to this work. many of these works were carried out under vacuum with precise control of physical separations between objects. ${ }^{4,5}$ Geometric optics have been widely used for imaging but have generally been ignored for applications in radiative heat transfer.

It turns out that geometric optics have been the most practical method available to improve efficiency of solar thermal systems ${ }^{8,9}$ and solar energy harvesting ${ }^{10}$ in general. Non-imaging optical design such as concentrators are routinely used to enhance the amount of captured sunlight. ${ }^{11,12}$ The sun is a blackbody, albeit at much higher temperature than the Earth. Thus, it is conceivable that waste heat in the form of thermal radiation can be harvested from hot objects more effectively by using similar optical techniques. Here, we report on an experimental demonstration of using a non-imaging compound parabolic concentrator (CPC) designed to harness thermal radiation from hot objects. We designed and fabricated a CPC in the mid-infrared (MIR) regime that has a 1.4 times enhancement for a diffused thermal source. Then, we experimentally demonstrated a consistent temperature rise by as much as $20{ }^{\circ} \mathrm{C}$ when the $\mathrm{CPC}$ is placed in between a heat source and a receiver. At the same time, we found via simulation and experiment that just concentrating heat flux can improve a thermoelectric (TE) module's efficiency by more than 1.5 times. Our method is non-contact, requires no moving parts, can work at a distance and does not occupy much physical space compared to heat exchanger systems.

\section{Simulation and Experimental Setup of Non- imaging Optics}

In order to use the design for collection in the mid-infrared, we decide to use a hollow design instead of IR transparent materials such as zinc selenide. Non-hollow design will naturally be subjected to more losses due to reflection and material absorption. However, there is a trade-off between the size of the CPC versus the collection efficiency. ${ }^{11}$ 
Furthermore, the manufacturing process of creating a reflective CPC with optically smooth surface and precise curvature is easily achievable, thus some sacrifices are needed such as restriction on minimum length of the diameter of the exit and entrance apertures and the height of the CPC. To maintain the large geometric concentration ratio with a relatively large acceptance angle, the exit aperture is set to be as small as $2 \mathrm{~mm}$. The acceptance angle is set to be 17.5 degrees, which will result the entrance aperture to be $6.66 \mathrm{~mm}$ to the height of $13.7 \mathrm{~mm}$ (see Fig. 1(a)). With these parameters, the geometric concentration ratio is calculated to be 11 , based on a collimated source which emitted parallel rays like the sun. ${ }^{112}$ The CPC was custom made by Delta Optics Technologies Ltd., ${ }^{13}$ involving diamond turn polishing to ensure good surface quality and gold coating of the inner surface.

With the custom-designed CPC, we would like to know how well our design performs under a constant thermal radiation emitted from a heat source. This was carried out using a ray-tracing program ZEMAX. $^{14}$ Most simulations of CPCs were conducted using sources emitting parallel rays so as to simulate the sun. However, waste heat from hot bodies around us are much smaller and much closer to us than the Sun, thus the emission will be more diffuse similar to a Lambertian source. Rather than using a collimated source, we have decided to use a Lambertian source which represents the thermal emitter more accurately. Note that we purposely chose a CPC with a relatively large acceptance angle which has been optimized for the range of separation $(20-80 \mathrm{~cm})$ between source and emitter. Fig. 1 (b) shows the simulated power received at the detector as a function of separation. We note that the received power is consistently higher with the CPC than without. Furthermore, the received power is consistently higher at all separations. However, we note that a concentration ratio of 11 is only valid for a collimated source. Under a diffuse illumination, we only achieved about $40 \%$ enhancement in power received and this enhancement decreases with increasing separation of the source from the emitter (Fig. 1(b)).

Next, we perform an experiment in a setup illustrated in Fig. 2(a). In this experiment, we used a 12 by $12 \mathrm{~cm}$ black ceramic heater placed a distance away from a thermocouple with and without the presence of a CPC. The temperature of the heater is measured to be $400{ }^{\circ} \mathrm{C}$ after

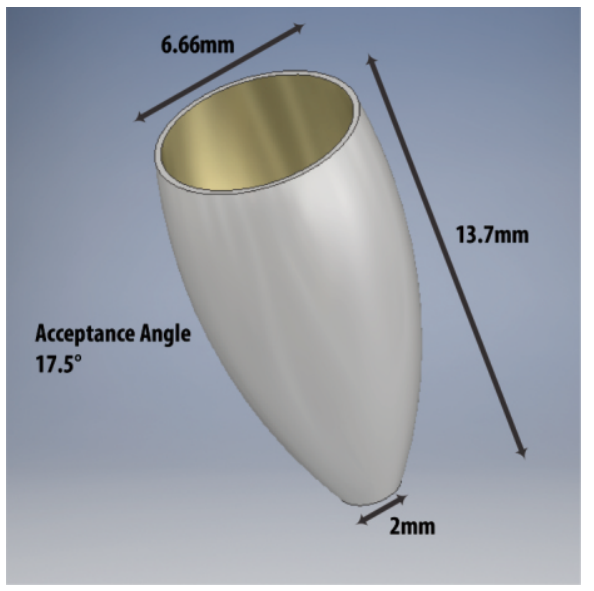

(a)

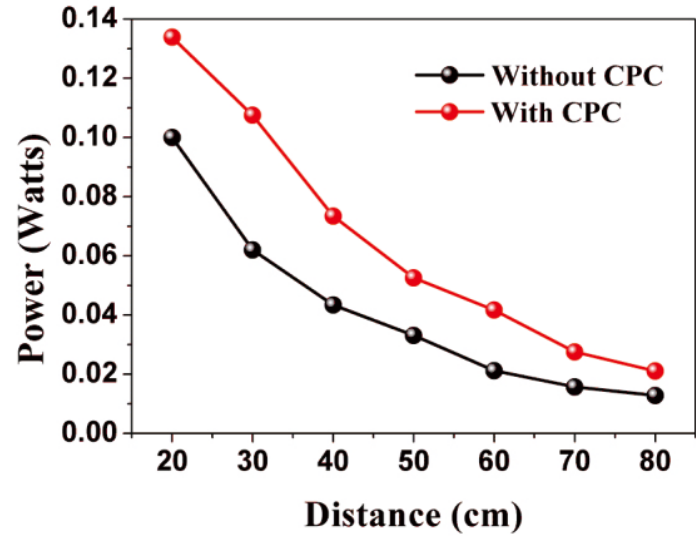

(b)

Fig. 1 (a) Schematic of the simulated compound parabolic concentrator (CPC). The acceptance angle is designed to be at $17.5^{\circ}$. The concentration ratio is around to 11. (b) Simulation results of received power using ZEMAX. The source is a 12 by $12 \mathrm{~cm}$ random emitter mimicking a blackbody and the receiver is 1 by $1 \mathrm{~mm}$. The heat source is modeled as a randomize diffuse source emitting at 1 Watt of optical power.

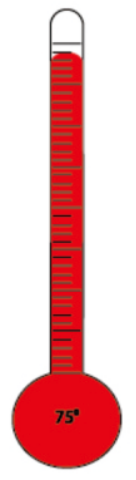

Receiver

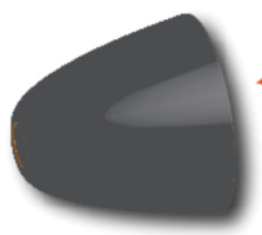

CPC

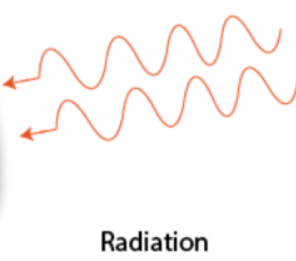

(a)

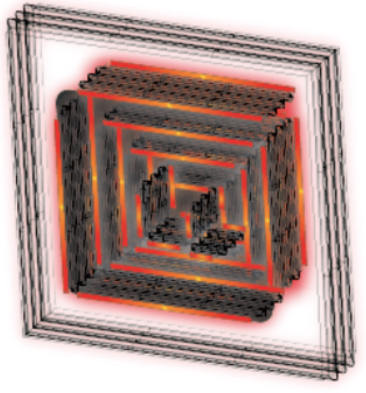

Heat source

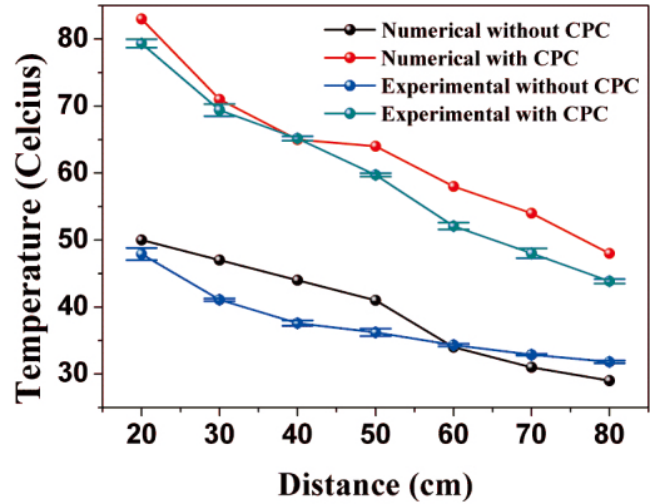

(b)

Fig. 2 (a) Schematic of experiment setup to test collection of radiation waste heat. A black heated plate stabilized around $400{ }^{\circ} \mathrm{C}$ is directed towards a point receiver which is a thermocouple. The thermocouple is shielded from other radiation sources. The entire setup is enclosed but the compartment is not purged not made into vacuum to mimic applications under ambient condition. (b) The comparison between the numerical results and the experimental results of the temperature change of receiver after using CPC. The case with CPC is always higher than the case without CPC in both cases. 
temperature is stabilized. The experiment is carried out under ambient conditions within an enclosure. The CPC will be placed right in front of the detector and the separation between the detector and the source is varied. The measurement process is performed by placing and removing the CPC from the detector for about 1 minute each, this whole process will be repeated for about 5 times to ensure that the results received remain constant. The CPC is occasionally removed from the path and put back into the path. We can see that the drop in temperature is concurrent and almost instantaneously correlated with the removal of the CPC. Likewise, the rise in temperature is also instantaneous.

In order to verify the reliability of the experimental results, numerical analysis is carried out by using the commercial software Fluent based on fluid computational analysis. According to the calculation of heat transfer based on blackbody radiation, we have calculated the proportion of thermal radiation in total heat transfer under natural ambient condition is $40 \%$. Therefore, we assumed the diffuse source as the blackbody emitter in simulation calculation. In the simulation process, the simulation of temperature change caused by using CPC was realized though changing the temperature of diffuse source which attained according to the simulation results of ZEMAX in the optical part ( the results shown in the Fig 1 (b)), so as to better compare the simulation results with the experimental results. The comparison between the numerical results and the experimental results is shown in Fig. 2 (b). We chose a blackbody radiation plate with the temperature of $400{ }^{\circ} \mathrm{C}$ as the heat source and its size is $12 \times 12 \mathrm{~cm}$, and the receiver is 1 by $1 \mathrm{~mm}$. The emissivity of the heat source is 1 and the temperature is kept constant during the simulation process. Compared to the size of heat source, a large enough space is chosen as the calculation area mimic the actual experiment. While the boundary condition of the calculation area is constant at room temperature, and there is no air flow in the process. We adjust the temperature of the heat source according to the change of radiation power to simulate the temperature change of the receiver after using the CPC. Fig. 2 (b) shows that the numerical results are close to the experimental results, the maximum relative deviation is less than $12 \%$, and the results present the effect of CPC on gathering energy and increasing surface temperature of objects. Also, the trend of the distance dependence follows our simulated trend in Fig. 1 (b). This implies that our simulation with a diffused source is a good representation of the experiment.

\section{Improving Thermoelectric Device Performance with CPC}

Thermoelectric devices rely on the Seebeck effect to convert thermal energy into electrical energy. The Seebeck effect occurs when two different conductors are connected together to form a loop and when a temperature difference is applied between the two nodes. Then the voltage is generated. Inside the thermoelectric device, the thermoelectric semiconductor $\mathrm{p}-\mathrm{n}$ junction is connected by a copper strip to form a current loop. The p-n junction array is typically placed between two alumina ceramic plates to provide mechanical support for the overall structure and to ensure electrical isolation of the array from other conductive surfaces, as shown in Fig. 3. During operation, the thermoelectric device is connected to an external load, and the external load must match the internal resistance of the thermoelectric module to achieve maximum power output and thermoelectric conversion efficiency. ${ }^{15-18}$

To validate our thermoelectric simulations, we modified our current design in Fig. 1 (a) by making a rectangular CPC. This is to ensure that we have a large enough exit aperture for testing with a commercial thermoelectric module and to fabricate the $\mathrm{CPC}$ using $3 \mathrm{D}$ printing and vacuum deposited metal methods. To verify the effectiveness of $\mathrm{CPC}$, the numerical simulation and experiment were carried out, based on the two structure of TEG1-287 commercialized $\mathrm{Bi}_{2} \mathrm{Te}_{3}$ thermoelectric module, defined as Model 1 (without CPC) and Model 2 (with CPC), respectively. Compared to Model 1, Model 2 has a considerable reduction in structure size and material consumption, and the comparison is shown in Table 1. Thus, the effect of CPC can be well verified in the improvement of thermoelectric performance.

\subsection{Experimental Results}

The infrared radiation energy of CPC is used to increase the surface temperature of the hot end of the thermoelectric device. For studying the influence of CPC on the improvement of thermoelectric performance under different working conditions, an experimental system was set up and schematic diagram is shown Fig. 4 (a), the CPC used in the experiment is shown in Fig. 4 (b). The modified CPC has an acceptance angle of 25 degrees. Six groups of heat source temperature were selected for research, including $100{ }^{\circ} \mathrm{C}, 150{ }^{\circ} \mathrm{C}, 200{ }^{\circ} \mathrm{C}, 250{ }^{\circ} \mathrm{C}$, $300{ }^{\circ} \mathrm{C}, 350{ }^{\circ} \mathrm{C}$, respectively and the cold end of TEG was maintained at $27^{\circ} \mathrm{C}$ during the experiment $\left(\mathrm{T}_{\mathrm{C}}=27^{\circ} \mathrm{C}\right)$.

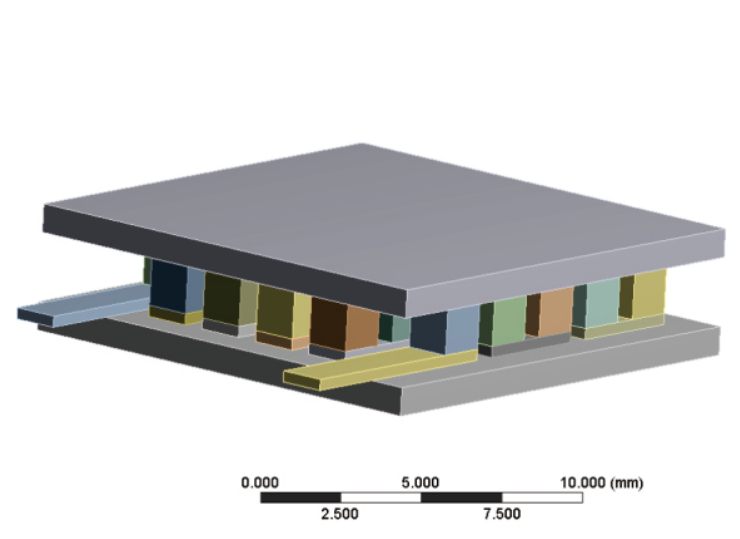

(a)

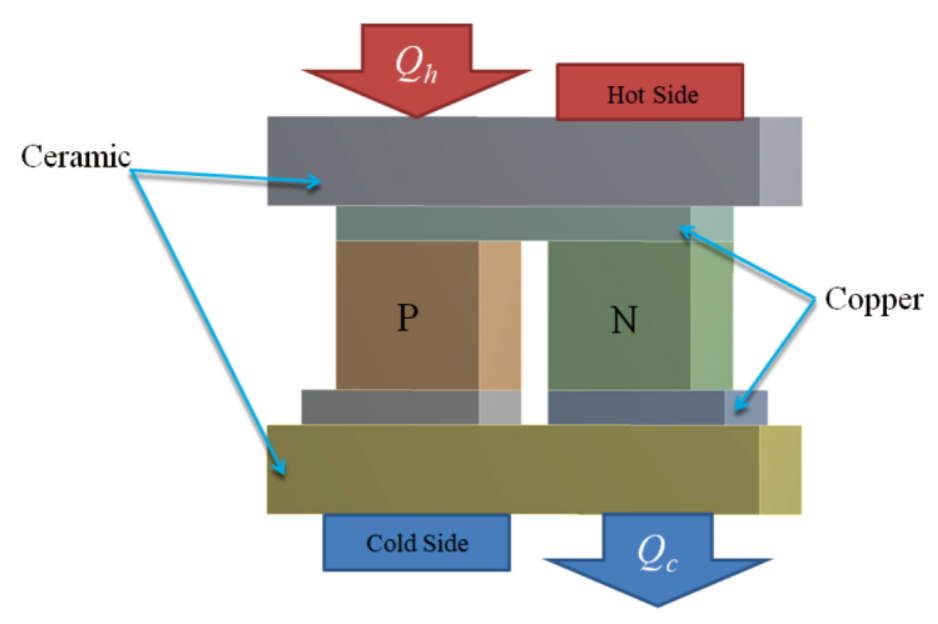

(b)

Fig. 3 (a) Geometry of the used thermoelectric (TE) (b) Schematic of unit of a p-n junction used as a thermoelectric. 
The experimental shows that the use of CPC can improve the thermoelectric performance under the condition of no air flow in the room and no other heat sources except nature heat dissipation into the air during the experiment.

The experiment system consists of the commercial TEG module, a copper plate heater, recycled water cooling system, an adjustable load resistor, and digital regulated DC power supply and data acquisition system as shown in Fig. 4 (a). The ceramic heating plate is powered by the DC power to form a heat source. The recycled water cooling system composed of a heat exchanger, a pump and constant temperature reservoir. The thermoelectric hot end receives the heat radiation energy from the heat source and uses the CPC to make energy easier gather and transfer centrally to hot end of thermoelectric module. The distance between the heat source and the TEG is $0.1 \mathrm{~m}$.

The device was insulated in cotton wrapping to isolate the effects of air and other thermal sources. We isolated the entire setup from the outside and only had one heat source to isolate the influence of other heat sources on the experimental results, which ensures that the radiated heat only acts on the hot end of the thermoelectric module. The temperature change during the experiment was recorded by high precision thermocouple and digital instrument. High thermal conductivity materials were used between the cold end of TEG and the

Table 1 Summary of geometric parameters.

\begin{tabular}{llccc}
\hline Geometric parameters & Symbol & Unit & \multicolumn{2}{c}{ Value } \\
\hline & & & Model 1 & Model 2 \\
\hline Number of thermocouple pairs per module & $n$ & $\backslash$ & 161 & 17 \\
Width of module & $W_{\text {TEM }}$ & $\mathrm{mm}$ & 40 & 15 \\
Length of module & $L_{\mathrm{TEM}}$ & $\mathrm{mm}$ & 40 & 15 \\
Internal resistance & $\backslash$ & $\Omega$ & 2.2 & 0.2 \\
\hline
\end{tabular}
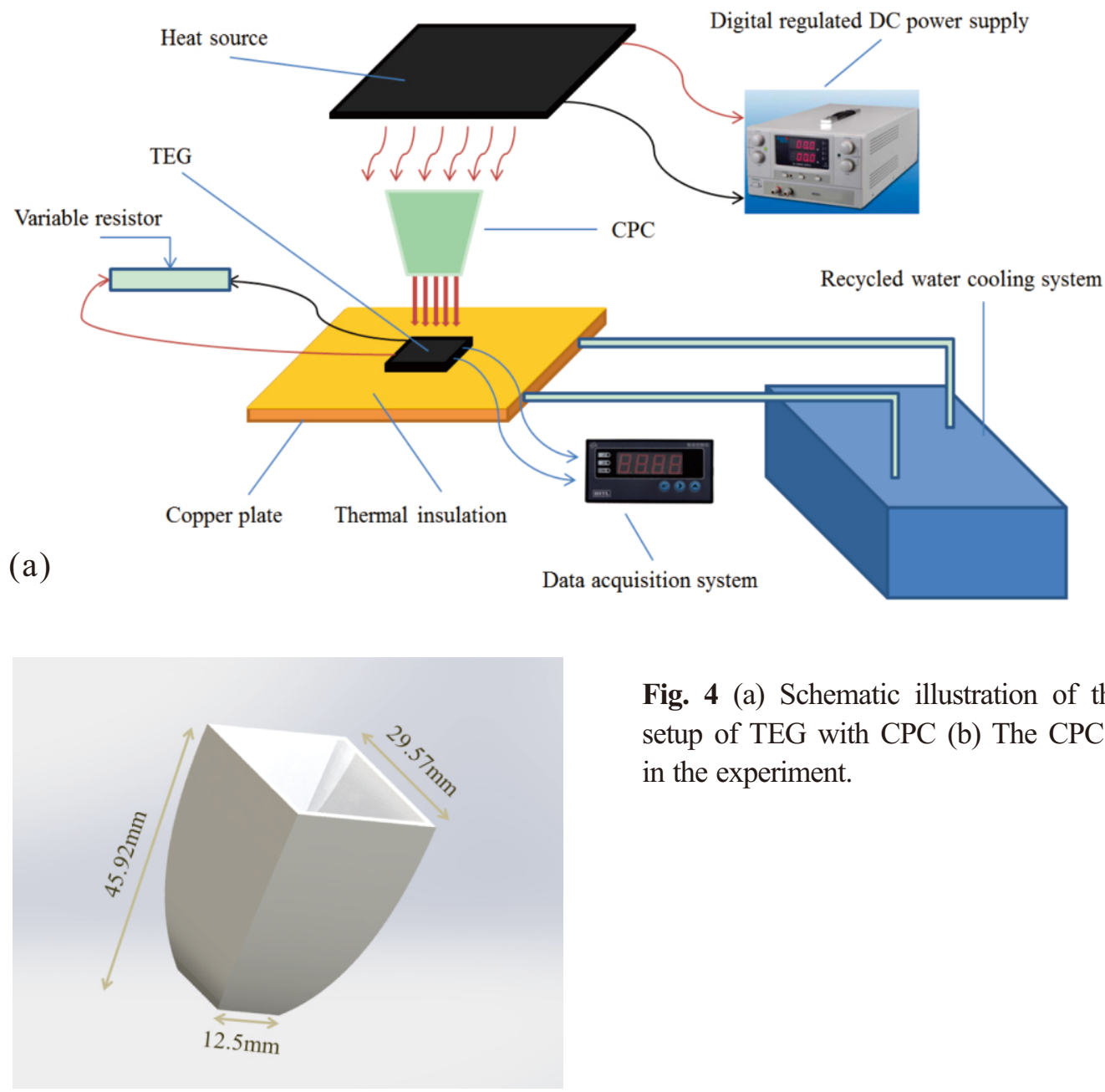

Fig. 4 (a) Schematic illustration of the experimental setup of TEG with CPC (b) The CPC model adopted in the experiment.

(b) 
copper plate to reduce the thermal resistance of the interface. The use of the recycled water cooling system keeps the temperature of the cold end at $27{ }^{\circ} \mathrm{C}$.

\subsection{Results and discussion}

To verify the effect of CPC energy concentration, numerical analysis is carried out by using the commercial software ANSYS based on finite element method. ${ }^{19-22}$ The governing equations, boundary conditions and material's properties (including thermal conductivity, electrical conductivity, and Seebeck coefficient) were referred to Ref. [19]. The temperature parameters of the cold and hot ends of TEG used in the simulation are determined by experiments.

In the calculation, the temperature distribution of TEG is shown in Fig. 5. It can be seen that the internal temperature drop mainly occurs is the thermal elements. Due to the temperature difference between the hot end and the cold end of TEG, the voltage was generated inside the module. The surface temperature of the hot end of Model 2 increases obviously after the CPC energy accumulation, which greatly enhances the temperature difference between the cold end and hot end of the thermoelectric device, and that of the main reason for the performance improvement of thermoelectric devices.

In this paper, two models of TEG structure are selected for performance test. Compared to Model 1, the Model 2 reduces greatly in structure size and number of PN junctions. In order to prove that the effect of $\mathrm{CPC}$ in improving the performance and optimizing the structure size of TEG, under the condition when the load resistance corresponds to the maximum power and efficiency of the two models, the Model 2 uses CPC to improve output performance and compares with Model 1 (without CPC). This test is beneficial for the exploration of the effective utilization of the waste heat recovered from CPC.

Fig. 6 is a graph showing the performance compassion between Model 1 without CPC and Model 2 with CPC. To study the influence of load resistance and temperature of heat source, the output power and conversion efficiency are defined as follows:

$$
P=U^{2} / R_{l}
$$

where $U$, and $R_{l}$ represent load resistance voltage and electric resistance, respectively, and the conversion efficiency can be calculated by:

$$
\eta=P / Q_{h}
$$

where $Q_{h}$ represents heat flow through the hot end of TEG. In order to calculate conversion efficiency more accurately, unlike the experiment described in Fig. 4 (a), the hot end of TEG was attached to the heat source, the cold end was attached to a copper water block with silicone tubes integrated for passing water through the block. The heat source and copper block were bolted together with the TEG in the middle, and each other of them coated the silicone grease to reduce contact thermal resistance. Externally we used heat insulation measures to ensure that all heat from the heat source is transferred to the hot end of TEG.

In general, when output power and efficiency is maximum, load resistance is close to the internal resistance of TEG. Consequently, we compare the output power and efficiency of Model 1 and Model 2 with the change of heat source temperature in the condition that the load resistance of Model 1 and Model 2 is close to the internal resistance (the load resistance of Model 1 is $2.2 \mathrm{ohms}$ and that Model 2 is $0.2 \mathrm{ohms}$ ).

As shown in Fig. 6 (a) and (b), with the increase of heat source temperature, the energy of heat source radiation increase gradually, which makes the surface temperature of hot end of TEG rise, the increase in temperature difference between the hot end and cold end of TEG causes the Seebeck effect of the thermoelectric material to be enhanced, thereby further increasing the output power and efficiency. As mentioned in this paper, the number of P-N junctions and structure size of Model 2 are less than those of Model 1, after using the CPC to gather energy, the output power and efficiency of the Model 2 are significantly improved. When the temperature of heat source is $100{ }^{\circ} \mathrm{C}$, the output power and efficiency of Model 1 are $0.0023 \mathrm{~W}, 0.08 \%$, respectively and that of Model 2 are $0.0076 \mathrm{~W}, 0.2 \%$, respective. The output power of Model 2 is up to 3.3 times higher than that of Model 1 and the efficiency is increased by a factor of 2.5 , and that of Model 2 with CPC have the greatest improvement over that of Model 1 without $\mathrm{CPC}$ than the other heat source temperature condition.

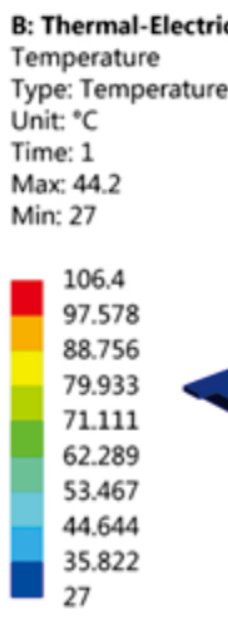

Model 1 without CPC

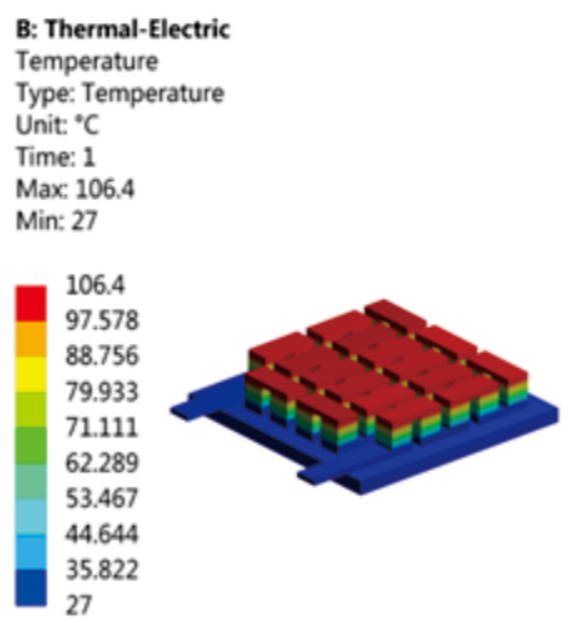

Model 2 with CPC

Fig. 5 Comparison of temperature contour of Model 1 and Model 2 at $350{ }^{\circ} \mathrm{C}$ of heat source temperature and $\mathrm{T}_{\mathrm{C}}=27^{\circ} \mathrm{C}$. 
Fig. 6 (c) and (d) presents the variation of output power and conversion efficiency of two kind of model with the change of load resistance under the condition of that the temperature of heat source is $350{ }^{\circ} \mathrm{C}$ and cold end of TEG is $27{ }^{\circ} \mathrm{C}$. It can be seen that the load resistance is close to the internal resistance of thermoelectric devices, the maximum output power and conversion efficiency are obtained and that increase first and the decrease with the increase of load resistance. The maximum output power and efficiency of Model 2 after using the CPC is $0.11 \mathrm{~W}$ and $0.902 \%$ respectively, and that of Model 1 without the CPC is $0.078 \mathrm{~W}$ and $0.4675 \%$ respectively, the output power of Model 2 is up to 1.41 times higher than that of Model 1 and the efficiency is almost increased by a factor of two. The experimental results show that the using of $\mathrm{CPC}$ into the TEG benefits the performance of thermoelectric device.

\section{Conclusion}

In this work, we demonstrated a non-imaging optical design capable to concentrating radiative waste heat which is a diffusive source. The simulated power received and measured temperature rise are in good agreement, with a $20{ }^{\circ} \mathrm{C}$ enhancement when non-imaging optics is used. Using the measured enhancement, we simulated and experimental measured the performance of a thermoelectric device with concentrated blackbody radiation. The results demonstrated great enhancement in both efficiency and output power, in which the output power is increased up to 1.41 times and the efficiency is almost increased by a factor of two. Our concept of using non-imaging optics for waste heat recovery is the first-of-its-kind that will bring a new paradigm for waste heat management without physical contact and moving parts. In the future work, we can adjust the size of thermoelectric device and CPC and the distance between them to design the array model according to the actual application, and make this novel topic not so far from being realized as a prototype.

\section{Acknowledgements}

This work is supported by the Basic Science Center Program for Ordered Energy Conversion of the National Nature Science Foundation of China (No. 51888103) and the CAS Pioneer Hundred Talents Program. D. D. acknowledges support by AME Young Individual Research Grants by the Agency for Science, Technology and Research under Grant No. A1884c0020. Y. Hao acknowledge the financial support from Chinese Academy of Sciences International Collaboration Key Program (182211KYSB20160043). We would like to thank Boon Ping Ng for his guidance in the ZEMAX simulations.

\section{References}

1. I. Johnson, W. T. Choate and A. Davidson, Technology and Opportunities in U.S. Industry, Tech. rep., BCS, Inc., Laurel, MD (United States), 2008, 3

2. H. Jouhara, N. Khordehgah, S. Almahmoud, B. Delpech, A. Chauhan and S. A. Tassou, Thermal Science and Engineering Progress, 2018, 6, 268-289.

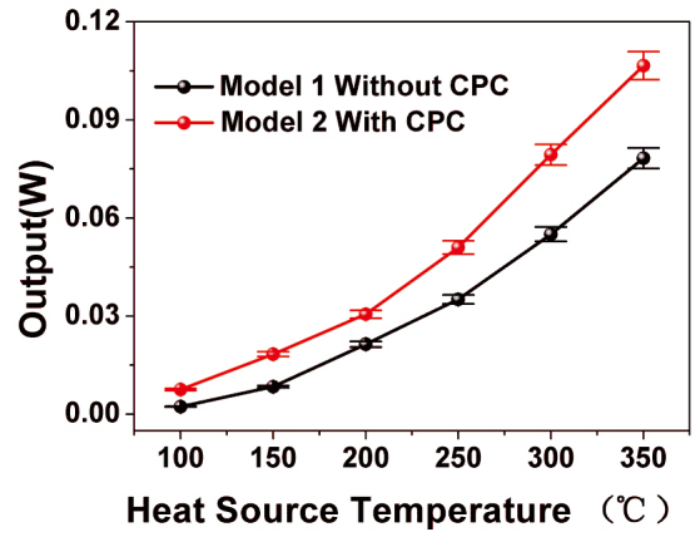

(a)

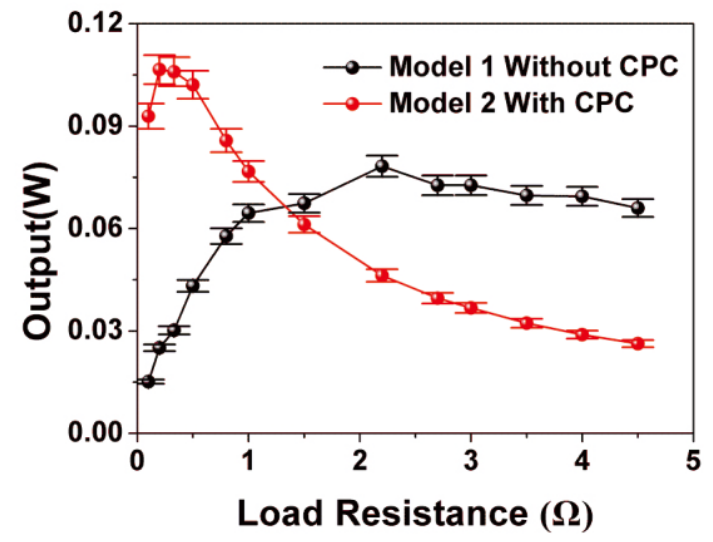

(c)

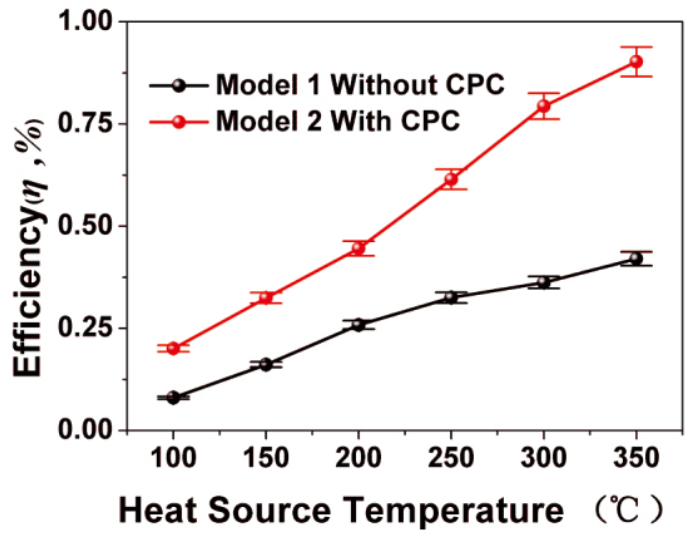

(b)

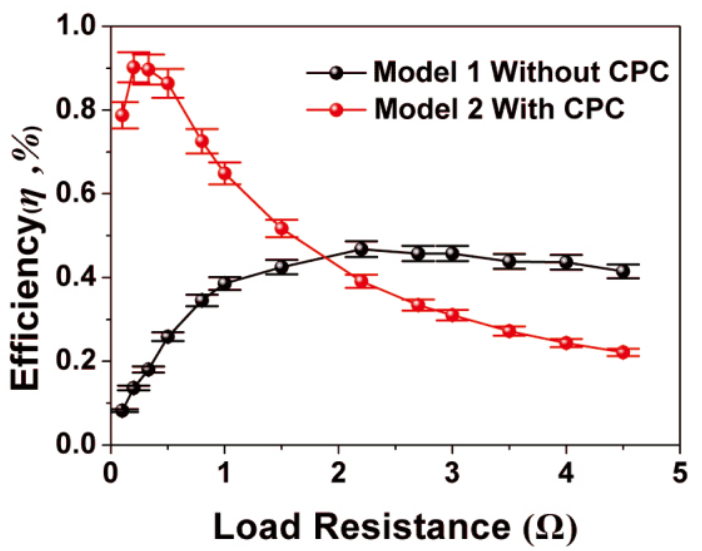

(d)

Fig. 6 The performance comparison between Model 1 and Model 2. In Figs. (a) and (b), the load resistance of Model 1 is 2.2 ohms and that Model 2 is $0.2 \mathrm{ohms}$; in Figs. (c) and (d), the temperature of heat source is $350{ }^{\circ} \mathrm{C}$ (a) Output power comparison with temperature of heat source (b) Efficiency compassion with temperature of heat source (c) Output power comparison with load resistance (d) Efficiency compassion with load resistance. 
3. B. A. Hedman, The EPA CHP Partnership: Waste Heat to Power 26.

4. Z. Yu, N. P. Sergeant, T. Skauli, G. Zhang, H. Wang and S. Fan, Nat. Commun., 2013, 4, 1730.

5. K. Kim, B. Song, V. Fernandez-Hurtado, W. Lee, W. Jeong, L. Cui, D. Thompson, J. Feist, M. T. Reid, F. J. Garcia-Vidal, J. C. Cuevas, E. Meyhofer and P. Reddy, Nature, 2015, 528, 387-391.

6. Y. Liu, F. Sun and S. He, Opt Express, 2016, 24, 5683-5692.

7. Y. M. Yao Zhai, Sabrina N. David, Dongliang Zhao, Runnan Lou, Gang Tan, Ronggui Yang and Xiaobo Yin, Science, 2017, aai7899

8. L. A. Weinstein, J. Loomis, B. Bhatia, D. M. Bierman, E. N. Wang and G. Chen, Chem. Rev., 2015, 115, 12797-12838.

9. T. T. Zhu, Y. H. Diao, Y. H. Zhao and F. F. Li, Appl. Therm. Eng., 2016, 98, 1201-1213.

10. I. Santos-González, M. Sandoval-Reyes, O. García-Valladares, N. Ortega and V. H. Gómez, Energy Procedia, 2014, 57, 2956-2965.

11. A. RABL, Sol. Energy, 1975, 18, 93-111.

12. A. RABL, Sol. Energy, 1975, 18, 497-511.

13. Home. http://deltaoptics.com/.

14. Leading Optical Product Design Software for Engineering TeamsZemax. https://www.zemax.com
15. Y. Deng, W. Zhu, Y. Wang and Y. Shi, Sol. Energy, 2013, 88, 182191.

16. O. M. Al-Habahbeh, A. Mohammad, A. Al-khalidi, M. Khanfer and M. Obeid, Journal of King Saud University - Engineering Sciences, 2018, 30 (2), 177-182.

17. Y. X. Zhen, M. Yang, H. Zhang and G. S. Fu, Sci. Bull., 2017, 62 (22), 1530-1537.

18. A. Eldesoukey and H. Hassan, Energ. Convers. Manage., 2019, 180, 231-239.

19. E. E. Antonova and D. C. Looman, ICT 2005. 24th International Conference on Thermoelectrics, 2005, 215-218.

20. M. J. Huang, R. H. Yen and A. B. Wang, Int. J. Heat Mass Tran, 2005, 48 (2), 413-418.

21. M. Chen, L. A. Rosendahl and T. Condra, Int. J. Heat Mass Tran., 2011, 54 (1), 345-355.

22. M. Liao, Z. He, C. Jiang, X. Fan, Y. Li and F. Qi, Appl. Therm. Eng., 2018, 133, 493-500.

Publisher's Note Engineered Science Publisher remains neutral with regard to jurisdictional claims in published maps and institutional affiliations. 